\title{
Extremal problems for $t$-partite and $t$-colorable hypergraphs
}

\author{
Dhruv Mubayi * John Talbot $^{\dagger}$ \\ Submitted: Aug 23, 2007; Accepted: Jan 20, 2008; Published: Feb 4, 2008 \\ Mathematics Subject Classification: 05D05
}

\begin{abstract}
Fix integers $t \geq r \geq 2$ and an $r$-uniform hypergraph $F$. We prove that the maximum number of edges in a $t$-partite $r$-uniform hypergraph on $n$ vertices that contains no copy of $F$ is $c_{t, F}\left(\begin{array}{l}n \\ r\end{array}\right)+o\left(n^{r}\right)$, where $c_{t, F}$ can be determined by a finite computation.

We explicitly define a sequence $F_{1}, F_{2}, \ldots$ of $r$-uniform hypergraphs, and prove that the maximum number of edges in a $t$-chromatic $r$-uniform hypergraph on $n$ vertices containing no copy of $F_{i}$ is $\alpha_{t, r, i}\left(\begin{array}{l}n \\ r\end{array}\right)+o\left(n^{r}\right)$, where $\alpha_{t, r, i}$ can be determined by a finite computation for each $i \geq 1$. In several cases, $\alpha_{t, r, i}$ is irrational. The main tool used in the proofs is the Lagrangian of a hypergraph.
\end{abstract}

\section{Introduction}

An $r$-uniform hypergraph or $r$-graph is a pair $G=(V, E)$ of vertices, $V$, and edges $E \subseteq\left(\begin{array}{l}V \\ r\end{array}\right)$, in particular a 2 -graph is a graph. We denote an edge $\left\{v_{1}, v_{2}, \ldots, v_{r}\right\}$ by $v_{1} v_{2} \cdots v_{r}$. Given $r$-graphs $F$ and $G$ we say that $G$ is $F$-free if $G$ does not contain a copy of $F$. The maximum number of edges in an $F$-free $r$-graph of order $n$ is $\operatorname{ex}(n, F)$. For $r=2$ and $F=K_{s}(s \geq 3)$ this number was determined by Turán [T41] (earlier Mantel [M07] found $\left.\operatorname{ex}\left(n, K_{3}\right)\right)$. However in general (even for $r=2$ ) the problem of determining the exact value of $\operatorname{ex}(n, F)$ is beyond current methods. The corresponding asymptotic problem is to determine the Turán density of $F$, defined by $\pi(F)=\lim _{n \rightarrow \infty} \frac{\operatorname{ex}(n, F)}{\left(\begin{array}{l}n \\ r\end{array}\right)}$ (this always exists by a simple averaging argument due to Katona et al. [KNS64]). For 2-graphs the Turán

*Department of Mathematics, Statistics and Computer Science, University of Illinois, Chicago, IL 60607, and Department of Mathematical Sciences, Carnegie-Mellon University, Pittsburgh, PA 15213. Email: mubayi@math.uic.edu. Research supported in part by NSF grants DMS-0400812 and 0653946 and an Alfred P. Sloan Research Fellowship.

${ }^{\dagger}$ Department of Mathematics, UCL, London, WC1E 6BT, UK. Email: talbot@math.ucl.ac.uk. This author is a Royal Society University Research Fellow. 
density is determined by the chromatic number of the forbidden subgraph $F$. The explicit relationship is given by the following fundamental result.

Theorem 1 (Erdös-Stone-Simonovits [ES46], [ES66]). If $F$ is a 2-graph then $\pi(F)=1-\frac{1}{\chi(F)-1}$.

When $r \geq 3$, determining the Turán density is difficult, and there are only a few exact results. Here we consider some closely related hypergraph extremal problems. Call a hypergraph $H t$-partite if its vertex set can be partitioned into $t$ classes, such that every edge has at most one vertex in each class. Call $H t$-colorable, if its vertex set can be partitioned into $t$ classes so that no edge is entirely contained within a class.

Definition 2. Fix $t, r \geq 2$ and an $r$-graph $F$. Let $\operatorname{ex}_{t}^{*}(n, F)\left(e x_{t}(n, F)\right)$ denote the maximum number of edges in a t-partite (t-colorable) $r$-graph on $n$ vertices that contains no copy of $F$. The t-partite Turán density of $F$ is $\pi_{t}^{*}(F)=\lim _{n \rightarrow \infty} e x_{t}^{*}(n, F) /\left(\begin{array}{l}n \\ r\end{array}\right)$ and the t-chromatic Turán density of $F$ is $\pi_{t}(F)=\lim _{n \rightarrow \infty} e x_{t}(n, F) /\left(\begin{array}{l}n \\ r\end{array}\right)$.

Note that it is easy to show that these limits exist. In this paper, we determine $\pi_{t}^{*}(F)$ for all $r$-graphs $F$ and determine $\pi_{t}(F)$ for an infinite family of $r$-graphs (previously no nontrivial value of $\pi_{t}(F)$ was known). In many cases our examples yield irrational values of $\pi_{t}(F)$. For the usual Turán density, $\pi(F)$ has not been proved to be irrational for any $F$, although there are several conjectures stating irrational values.

In order to describe our results, we need the concept of $G$-colorings which we introduce now. If $F$ and $G$ are hypergraphs (not necessarily uniform) then $F$ is $G$-colorable if there exists $c: V(F) \rightarrow V(G)$ such that $c(e) \in E(G)$ whenever $e \in E(F)$. In other words, $F$ is $G$-colorable if there is a homomorphism from $F$ to $G$.

Let $K_{t}^{(r)}$ denote the complete $r$-graph of order $t$. Then an $r$-graph $F$ is $t$-partite if $F$ is $K_{t}^{(r)}$-colorable, and $F$ is $t$-colorable if it is $H_{t}^{(r)}$-colorable where $H_{t}^{(r)}$ is the (in general nonuniform) hypergraph consisting of all subsets $A \subseteq\{1,2, \ldots, t\}$ satisfying $2 \leq|A| \leq r)$. The chromatic number of $F$ is $\chi(F)=\min \{t \geq 1: F$ is $t$-colorable $\}$. Note that while a 2 -graph is $t$-colorable iff it is $t$-partite this is no longer true for $r \geq 3$, for example $K_{4}^{(3)}$ is 2-colorable but not 2-partite or 3-partite.

Let $\mathcal{G}_{t}^{(r)}$ denote the collection of all $t$-vertex $r$-graphs with vertex $\{1,2, \ldots, t\}$. A tool which has proved very useful in extremal graph theory and which we will use later is the Lagrangian of an $r$-graph. Let

$$
\mathbb{S}_{t}=\left\{\vec{x} \in \mathbb{R}^{t}: \sum_{i=1}^{t} x_{i}=1, x_{i} \geq 0 \text { for } 1 \leq i \leq t\right\} .
$$

If $G \in \mathcal{G}_{t}^{(r)}$ and $\vec{x} \in \mathbb{S}_{t}$ then we define

$$
\lambda(G, \vec{x})=\sum_{v_{1} v_{2} \cdots v_{r} \in E(G)} x_{v_{1}} x_{v_{2}} \cdots x_{v_{t}} .
$$

The Lagrangian of $G$ is $\max _{\vec{x} \in \mathbb{S}_{t}} \lambda(G, \vec{x})$. The first application of the Lagrangian to extremal graph theory was due to Motzkin and Strauss who gave a new proof of Turán's theorem. We are now ready to state our main result. 
Theorem 3. If $F$ is an $r$-graph and $t \geq r \geq 2$ then

$$
\pi_{t}^{*}(F)=\max \left\{r ! \lambda(G): G \in \mathcal{G}_{t}^{(r)} \text { and } F \text { is not } G \text {-colorable }\right\} .
$$

As an example of Theorem 3, suppose that $t=4, r=3$, and $F=K_{4}^{(3)}$. Let $H$ denote the unique 3 -graph with four vertices and three edges. Now $F$ is $F$-colorable, but it is not $H$-colorable, and the Lagrangian $\lambda(H)$ of $H$ is $4 / 81$, achieved by assigning the degree three vertex a weight of $1 / 3$ and the other three vertices a weight of $2 / 9$. Consequently, Theorem 3 says that the maximum number of edges in an $n$-vertex 4 -partite 3 -graph containing no copy of $K_{4}^{(3)}$ is $(8 / 27)\left(\begin{array}{l}n \\ 3\end{array}\right)+o\left(n^{3}\right)$. This is clearly achievable, by the 4partite 3 -graph with part sizes $n / 3,2 n / 9,2 n / 9,2 n / 9$, with all possible triples between three parts that include the largest (of size $n / 3$ ), and no triples between the three small parts.

Chromatic Turán densities were previously considered in [T07] where they were used to give an improved upper bound on $\pi(H)$, where $H$ is defined in the previous paragraph. However no non-trivial chromatic Turán densities have previously been determined. For each $r \geq t \geq 2$ we are able to give an infinite sequence of $r$-graphs whose $t$-chromatic Turán densities are determined exactly.

For $l \geq t \geq 2$ and $r \geq 2$ define

$$
\beta_{r, t, l}:=\max \{\lambda(G): G \text { is a } t \text {-colorable } r \text {-graph on } l \text { vertices }\} \text {. }
$$

It seems obvious that $\beta_{r, t, l}$ is achieved by the $t$-chromatic $r$-graph of order $l$ with all color classes of size $\lfloor l / t\rfloor$ or $\lceil l / t\rceil$ and all edges present except those within the classes. Note that if $t \mid l$ then this would give

$$
\beta_{r, t, l}=\left(\left(\begin{array}{l}
l \\
r
\end{array}\right)-t\left(\begin{array}{c}
l / t \\
r
\end{array}\right)\right) \frac{1}{l^{r}}
$$

However, we are only able to prove this for $r=2,3$. If the above statement is true, then $\beta_{r, t, l}$ can be computed by calculating the maximum of an explicit polynomial in one variable over the unit interval. In any case it can be obtained by a finite computation (for fixed $r, t, l)$. Let $\alpha_{r, t, l}=r ! \beta_{r, t, l}$.

Theorem 4. Fix $l \geq r \geq 2$. Let $L_{l+1}^{(r)}$ be the r-graph obtained from the complete graph $K_{l+1}$ by enlarging each edge with a set of $r-2$ new vertices. If $t \geq 2$ then

$$
\pi_{t}\left(L_{l+1}^{(r)}\right)=\alpha_{r, t, l}
$$

where $\alpha_{r, t, l}$ is defined above.

The remainder of the paper is arranged as follows. In the next section we prove Theorem 3 and in the last section we prove Theorem 4 and the statements about computing $\beta_{r, t, l}$, for $r=2,3$. 


\section{Proof of Theorem 3}

If $G \in \mathcal{G}_{t}^{(r)}$ and $\vec{x}=\left(x_{1}, \ldots, x_{t}\right) \in \mathbb{Z}_{+}^{t}$ then the $\vec{x}$-blow-up of $G$ is the $r$-graph $G(\vec{x})$ constructed from $G$ by replacing each vertex $v$ by a class of vertices of size $x_{v}$ and taking all edges between any $r$ classes corresponding to an edge of $G$. More precisely we have $V(G(\vec{x}))=X_{1} \dot{\cup} \cdots \dot{\cup} X_{t},\left|X_{i}\right|=x_{i}$ and

$$
E(G(\vec{x}))=\left\{\left\{v_{i_{1}} v_{i_{2}} \cdots v_{i_{r}}\right\}: v_{i_{j}} \in X_{i_{j}},\left\{i_{1} i_{2} \cdots i_{r}\right\} \in E(G)\right\} .
$$

If $\vec{x}=(s, s, \ldots, s)$ and $G=K_{t}^{(r)}$ then $G(\vec{x})$ is the complete $t$-partite $r$-graph with class size $s$, denoted by $K_{t}^{(r)}(s)$. Note that if $F$ and $G$ are both $r$-graphs then $F$ is $G$-colorable iff there exists $\vec{x} \in \mathbb{Z}_{+}^{t}$ such that $F \subseteq G(\vec{x})$.

An $r$-graph $G$ is said to be covering if each pair of vertices in $V(G)$ is contained in a common edge. If $W \subset V$ and $G$ is an $r$-graph with vertex $V$ then $G[W]$ is the induced subgraph of $G$ formed by deleting all vertices not in $W$ and removing all edges containing these vertices.

Lemma 5 (Frankl and Rödl [FR84]). If $G$ is an r-graph of order $n$ then there exists $\vec{y} \in \mathbb{S}_{n}$ with $\lambda(G)=\lambda(G, \vec{y})$, such that if $P=\left\{v \in V(G): y_{v}>0\right\}$ then $G[P]$ is covering.

Supersaturation for ordinary Turán densities was shown by Erdős [E71]. The proof for $G$-chromatic Turán densities is essentially identical but for completeness we give it. We require the following classical result.

Theorem 6 (Erdös [E64]). If $r \geq 2$ and $t \geq 1$ then $\operatorname{ex}\left(n, K_{r}^{(r)}(t)\right)=O\left(n^{r-\lambda_{r, t}}\right)$, with $\lambda_{r, t}>0$.

Lemma 7 (Supersaturation). Fix $t \geq r \geq 2$. If $G$ is an $r$-graph, $\mathcal{H}$ is a finite family of $r$-graphs, $s \geq 1$ and $\vec{s}=(s, s, \ldots, s)$ then $\pi_{t}^{*}(\mathcal{H}(\vec{s}))=\pi_{t}^{*}(\mathcal{H})$ (where $\mathcal{H}(\vec{s})=\{H(\vec{s})$ : $H \in \mathcal{H}\}$ ).

Proof: Let $p=\max \{|V(H)|: H \in \mathcal{H}\}$. By adding isolated vertices if necessary we may suppose that every $H \in \mathcal{H}$ has exactly $p$ vertices.

First we claim that if $F$ is an $n$-vertex $r$-graph with density at least $\alpha+2 \epsilon$, where $\alpha, \epsilon>0$, and $r \leq m \leq n$ then at least $\epsilon\left(\begin{array}{c}n \\ m\end{array}\right)$ of the $m$-vertex induced subgraphs of $F$ have density at least $\alpha+\epsilon$. To see this note that if it fails to hold then

$$
\left(\begin{array}{c}
n-r \\
m-r
\end{array}\right)(\alpha+2 \epsilon)\left(\begin{array}{c}
n \\
r
\end{array}\right) \leq \sum_{W \in\left(\begin{array}{c}
V(F) \\
m
\end{array}\right)} e(F[W])<\epsilon\left(\begin{array}{c}
n \\
m
\end{array}\right)\left(\begin{array}{c}
m \\
r
\end{array}\right)+(1-\epsilon)\left(\begin{array}{c}
n \\
m
\end{array}\right)(\alpha+\epsilon)\left(\begin{array}{c}
m \\
r
\end{array}\right),
$$

which is impossible.

Let $\epsilon>0$ and suppose that $F$ is a $t$-partite $n$-vertex $r$-graph with density at least $\pi_{t}^{*}(\mathcal{H})+2 \epsilon$. We need to show that if $n$ is sufficiently large then $F$ contains a copy of $\mathcal{H}(\vec{s})$. Let $m \geq m(\epsilon)$ be sufficiently large that any $t$-partite $m$-vertex $r$-graph with density at least $\pi_{t}^{*}(\mathcal{H})+\epsilon$ contains a copy of some $H \in \mathcal{H}$. We say that $W \in\left(\begin{array}{c}V(F) \\ m\end{array}\right)$ is good if 
$F[W]$ contains a copy of some $H \in \mathcal{H}$. By the claim at least $\epsilon\left(\begin{array}{c}n \\ m\end{array}\right) m$-sets are good, so if $\delta=\epsilon /|\mathcal{H}|$ then at least $\delta\left(\begin{array}{l}n \\ m\end{array}\right) m$-sets contain a fixed $H^{*} \in \mathcal{H}$.

Thus the number of $p$-sets $U \subset V(F)$ such that $F[U] \simeq H^{*}$ is at least

$$
\frac{\delta\left(\begin{array}{c}
n \\
m
\end{array}\right)}{\left(\begin{array}{c}
n-p \\
m-p
\end{array}\right)}=\frac{\delta\left(\begin{array}{l}
n \\
p
\end{array}\right)}{\left(\begin{array}{c}
m \\
p
\end{array}\right)} .
$$

Let $J$ be the $p$-graph with vertex set $V(F)$ and edge set consisting of those $p$-sets $U \subset$ $V(F)$ such that $F[U] \simeq H^{*}$. Now, by Theorem 6 , $\operatorname{ex}_{t}^{*}\left(n, K_{p}^{(p)}(l)\right) \leq \operatorname{ex}\left(n, K_{p}^{(p)}(l)\right)=$ $O\left(n^{p-\lambda_{p, l}}\right)$, where $\lambda_{p, l}>0$. Hence (1) implies that for any $l \geq p$ if $n$ is sufficiently large then $K_{p}^{(p)}(l) \subset J$.

Finally consider a coloring of the edges of $K_{p}^{(p)}(l)$ with $p$ ! different colors, where the color of the edge is given by the order in which the vertices of $H^{*}$ are embedded in it. By Ramsey's theorem if $l$ is sufficiently large then there is a copy of $K_{p}^{(p)}(s)$ with all edges the same color. This yields a copy of $H^{*}(\vec{s})$ in $F$ as required.

Proof of Theorem 3. Let $\alpha_{r, t}=\max \left\{r ! \lambda(G): G \in \mathcal{G}_{t}^{(r)}\right.$ and $F$ is not $G$-colorable $\}$. (This is well-defined since $\left|\mathcal{G}_{t}^{(r)}\right| \leq 2^{\left(\begin{array}{l}t \\ r\end{array}\right)}$ is finite.)

If $G \in \mathcal{G}_{t}^{(r)}$ and $F$ is not $G$-colorable then for any $\vec{x} \in \mathbb{Z}_{+}^{t}$ we have $F \nsubseteq G(\vec{x})$. Let $\vec{y} \in \mathbb{S}_{t}$ satisfy $\lambda(G, \vec{y})=\lambda(G)$. For $n \geq 1$ let $\vec{x}_{n}=\left(\left\lfloor y_{1} n\right\rfloor, \ldots,\left\lfloor y_{t} n\right\rfloor\right) \in \mathbb{Z}_{+}^{t}$. If $G_{n}=G\left(\vec{x}_{n}\right)$ then

$$
\lim _{n \rightarrow \infty} \frac{e\left(G_{n}\right)}{\left(\begin{array}{l}
n \\
r
\end{array}\right)}=r ! \lambda(G) .
$$

Moreover since each $G_{n}$ is $F$-free, $t$-partite and of order at most $n$ we have $\pi_{t}^{*}(F) \geq r ! \lambda(G)$. Hence $\pi_{t}^{*}(F) \geq \alpha_{r, t}$.

Let $\mathcal{H}(F)=\left\{H \in \mathcal{G}_{t}^{(r)}: F\right.$ is $H$-colorable $\}$.

It is sufficient to show that

$$
\pi_{t}^{*}(\mathcal{H}(F)) \leq \alpha_{r, t}
$$

Indeed, if we assume that (2) holds, then let $s \geq 1$ be minimal such that every $H \in \mathcal{H}(F)$ satisfies $F \subseteq H(\vec{s})$, where $\vec{s}=(s, s, \ldots, s)$. (Note that $s$ exists since $F$ is $H$-colorable for every $H \in \mathcal{H}(F)$ ). Now by supersaturation (Lemma 7) if $\epsilon>0$, then any $t$-partite $r$-graph $G_{n}$ with $n \geq n_{0}(s, \epsilon)$ vertices and density at least $\alpha_{r, t}+\epsilon$ will contain a copy of $H(\vec{s})$ for some $H \in \mathcal{H}(F)$. In particular $G_{n}$ contains $F$ and so $\pi_{t}^{*}(F) \leq \alpha_{r, t}$.

Let $\pi_{t}^{*}(\mathcal{H}(F))=\gamma$ and $\epsilon>0$. If $n$ is sufficiently large there exists an $\mathcal{H}(F)$-free, $t$-partite $r$-graph $G_{n}$ of order $n$ satisfying

$$
\frac{r ! e\left(G_{n}\right)}{n^{r}} \geq \gamma-\epsilon
$$

Taking $\vec{y}=(1 / n, 1 / n, \ldots, 1 / n) \in \mathbb{S}_{n}$ we have

$$
r ! \lambda\left(G_{n}\right) \geq r ! \lambda\left(G_{n}, \vec{y}\right)=\frac{r ! e\left(G_{n}\right)}{n^{r}} \geq \gamma-\epsilon
$$


Now Lemma 5 implies that there exists $\vec{z} \in \mathbb{S}_{n}$ satisfying

- $\lambda\left(G_{n}\right)=\lambda\left(G_{n}, \vec{z}\right)$ and

- $G_{n}[P]$ is covering where $P=\left\{v \in V(G): z_{v}>0\right\}$.

Since $G_{n}$ is $t$-partite, we conclude that $G_{n}[P]$ has at most $t$ vertices. Moreover, $G_{n}$ is $\mathcal{H}(F)$-free and so $G_{n}[P] \notin \mathcal{H}(F)$. Thus $F$ is not $G_{n}[P]$-colorable, and we have $\gamma-\epsilon \leq$ $r ! \lambda\left(G_{n}[P]\right) \leq \alpha_{r, t}$. Thus $\pi_{t}^{*}(\mathcal{H}(F)) \leq \alpha_{r, t}+\epsilon$ for all $\epsilon>0$. Hence (2) holds and the proof is complete.

\section{Infinitely many chromatic Turán densities}

For $l, r \geq 2$ let $\mathcal{K}_{l}^{(r)}$ be the family of $r$-graphs with at most $\left(\begin{array}{l}l \\ 2\end{array}\right)$ edges that contain a set $S$, called the core, of $l$ vertices, with each pair of vertices from $S$ contained in an edge. Note that $L_{l+1}^{(r)} \in \mathcal{K}_{l+1}^{(r)}$. We need the following Lemma that was proved in [M06]. For completeness, we repeat the proof below.

Lemma 8. If $K \in \mathcal{K}_{l+1}^{(r)}, s=\left(\begin{array}{c}l+1 \\ 2\end{array}\right)+1$ and $\vec{s}=(s, s, \ldots, s)$ then $L_{l+1}^{(r)} \subseteq K(\vec{s})$.

Proof. We first show that $L_{l+1}^{(r)} \subset L\left(\left(\begin{array}{c}l+1 \\ 2\end{array}\right)+1\right)$ for every $L \in \mathcal{K}_{l+1}^{(r)}$. Pick $L \in \mathcal{K}_{l+1}^{(r)}$, and let $L^{\prime}=L\left(\left(\begin{array}{c}l+1 \\ 2\end{array}\right)+1\right)$. For each vertex $v \in V(L)$, suppose that the clones of $v$ are $v=v^{1}, v^{2}, \ldots, v^{\left(\begin{array}{c}l+1 \\ 2\end{array}\right)+1}$. In particular, identify the first clone of $v$ with $v$.

Let $S=\left\{w_{1}, \ldots, w_{l+1}\right\} \subset V(L)$ be the core of $L$. For every $1 \leq i<j \leq l+1$, let $E_{i j} \in L$ with $E_{i j} \supset\left\{w_{i}, w_{j}\right\}$. Replace each vertex $z$ of $E_{i j}-\left\{w_{i}, w_{j}\right\}$ by $z^{q}$ where $q>1$, to obtain an edge $E_{i j}^{\prime} \in L^{\prime}$. Continue this procedure for every $i, j$, making sure that whenever we encounter a new edge it intersects the previously encountered edges only in $L$. Since the number of clones is $\left(\begin{array}{c}l+1 \\ 2\end{array}\right)+1$, this procedure can be carried out successfully and results in a copy of $L_{l+1}^{(r)}$ with core $S$. Therefore $L_{l+1}^{(r)} \subset L^{\prime}=L\left(\left(\begin{array}{c}l+1 \\ 2\end{array}\right)+1\right)$. Consequently, Lemma 7 implies that $\pi\left(L_{l+1}^{(r)}\right) \leq \pi\left(\mathcal{K}_{l+1}^{(r)}\right)$.

Proof of Theorem 4. Let $l \geq r \geq 2$ and $t \geq 2$. We will prove that

$$
\pi_{t}\left(\mathcal{K}_{l+1}^{(r)}\right)=\alpha_{r, t, l}
$$

The theorem will then follow immediately from Lemmas 7 and 8 . Let

$$
\mathcal{B}_{r, t, l}=\left\{G: G \text { is a } t \text {-colorable } \mathcal{K}_{l+1}^{(r)} \text {-free } r \text {-graph }\right\}
$$

Claim. $\max \left\{\lambda(G): G \in \mathcal{B}_{r, t, l}\right\}=\beta_{r, t, l}=\alpha_{r, t, l} / r !$.

Proof of Claim. If $G \in \mathcal{B}_{r, t, l}$ has order $n$ then Lemma 5 implies that there is $\vec{y} \in \mathbb{S}_{n}$ such that $\lambda(G)=\lambda(G, \vec{y})$ with $G[P]$ covering, where $P=\left\{v \in V(G): y_{v}>0\right\}$. Since $G$ is $\mathcal{K}_{l+1}^{(r)}$-free, we conclude that $|P|=p \leq l$. Hence there is $H \in \mathcal{B}_{r, t, l}$ such that $\lambda(H)=\lambda(G)$ and $H$ has order at most $l$. Consequently, $\max \left\{\lambda(G): G \in \mathcal{B}_{r, t, l}\right\} \leq \beta_{r, t, l}$. For the other inequality, we just observe that an $l$-vertex $r$-graph must be $\mathcal{K}_{l+1}^{(r)}$-free. 
Now we can quickly complete the proof of the theorem by proving (3). For the upper bound, observe that if $G \in \mathcal{B}_{r, t, l}$ has order $n$ then by the Claim

$$
\frac{e(G)}{n^{r}} \leq \lambda(G) \leq \frac{\alpha_{r, t, l}}{r !}
$$

and so $\pi_{t}\left(\mathcal{K}_{l+1}^{(r)}\right) \leq \alpha_{r, t, l}$. For the lower bound, suppose that $G \in \mathcal{B}_{r, t, l}$ has order $p$ and satisfies $\lambda(G)=\beta_{r, t, l}$. Then there exists $\vec{y} \in \mathbb{S}_{p}$ such that $\lambda(G, \vec{y})=\lambda(G)=\beta_{r, t, l}$. For $n \geq p$ define $\vec{y}_{n}=\left(\left\lfloor y_{1} n\right\rfloor, \ldots,\left\lfloor y_{p} n\right\rfloor\right)$. Now $\left\{G\left(\vec{y}_{n}\right)\right\}_{n=p}^{\infty}$ is a sequence of $t$-colorable $\mathcal{K}_{l+1}^{(r)}$-free $r$-graphs and hence

$$
\pi_{t}\left(\mathcal{K}_{l+1}^{(r)}\right) \geq \lim _{n \rightarrow \infty} \frac{e\left(G_{n}\right)}{\left(\begin{array}{l}
n \\
r
\end{array}\right)}=r ! \lambda(G)=\alpha_{r, t, l}
$$

Now we prove that $\beta_{r, t, l}$ can be computed by only considering maximum $t$-colorable $r$-graphs with almost equal part sizes when $r=2,3$. The case $r=2$ follows trivially from Lemma 5 so we consider the case $r=3$.

Theorem 9. Fix $l \geq t \geq 2$. Then $\beta_{3, t, l}$ is achieved by the $t$-chromatic 3 -graph of order $l$ with all color classes of size $\lfloor l / t\rfloor$ or $\lceil l / t\rceil$ and all edges present except those within the classes.

Remark: Note that if $t \mid l$ then this implies that $\beta_{3, t, l}=\left(\left(\begin{array}{l}l \\ 3\end{array}\right)-t\left(\begin{array}{c}l / t \\ 3\end{array}\right)\right) \frac{1}{l^{3}}$.

Proof. Let $G$ be a $t$-chromatic 3 -graph of order $l$ satisfying $\lambda(G)=\beta_{3, t, l}$. We may suppose (by adding edges as required) that $V(G)=V_{1} \cup V_{2} \cup \cdots \cup V_{t}$ and that all edges not contained in any $V_{i}$ are present. We may also suppose that $\left|V_{1}\right| \geq\left|V_{2}\right| \geq \cdots \geq\left|V_{t}\right|$. Let $\vec{x} \in \mathbb{S}_{p}$ satisfy $\lambda(G, \vec{x})=\lambda(G)$.

If $v, w \in V_{i}$ and $x_{v}>x_{w}$ then setting $\delta=\left(x_{v}-x_{w}\right) / 2>0$ and defining a new weighting $\vec{x}^{\prime}$ by $x_{v}^{\prime}=x_{v}-\delta, x_{w}^{\prime}=x_{w}+\delta$ and $x_{u}^{\prime}=x_{u}$ for $u \in V \backslash\{v, w\}$ it is easy to check that $\lambda\left(G, \vec{x}^{\prime}\right)>\lambda(G, \vec{x})$, contradicting the assumption that $\lambda(G, \vec{x})=\lambda(G)$. Hence we may suppose that there are $x_{1}, \ldots, x_{t} \geq 0$ such that all vertices in $V_{i}$ receive weight $x_{i}$.

In fact we can assume that all the $x_{i}$ are non-zero. Since $\vec{x} \in \mathbb{S}_{p}$ there exists $k$ such that $x_{k}>0$. Suppose that $x_{j}=0$ for some $j \in\{1,2, \ldots, t\}$. Let $a_{k}=\left|V_{k}\right|, a_{j}=\left|V_{j}\right|$ and $\epsilon=x_{k} a_{j} a_{k} /\left(a_{j}+a_{k}\right)$. Define a new weighting $\vec{x}^{\prime \prime}$ by $x_{v}^{\prime \prime}=x_{v}$ for $v \in V \backslash\left(V_{k} \cup V_{j}\right)$, $x_{v}^{\prime \prime}=\epsilon / a_{j}$ for $v \in V_{j}$ and $x_{v}^{\prime \prime}=x_{k}-\epsilon / a_{k}$ for $v \in V_{k}$. It is straightforward to check that $\vec{x}^{\prime \prime} \in \mathbb{S}_{p}$ and $\lambda\left(G, \vec{x}^{\prime \prime}\right)>\lambda(G, \vec{x})$, contradicting the maximality of $\lambda(G, \vec{x})$. Hence we may suppose that all the $x_{i}$ are non-zero.

Let $l=b t+c, 0 \leq c<t$. To complete the proof we need to show that all of the $V_{i}$ have order $b$ or $b+1$. Suppose, for a contradiction, that there exist $V_{i}$ and $V_{j}$ with $a_{i}=\left|V_{i}\right|$, $a_{j}=\left|V_{j}\right|$ and $a_{i} \geq a_{j}+2$. We will construct a new $t$-colorable $l$-vertex 3 -graph $\tilde{G}$ with $\lambda(\tilde{G})>\lambda(G)$.

We construct $\tilde{G}$ from $G$ by moving a vertex $v$ from $V_{i}$ to $V_{j}$ and inserting all new allowable edges (i.e. those which contain $v$ and 2 vertices from $V_{i} \backslash\{v\}$ ) while deleting any 
edges which now lie in $V_{j}$. By our assumption that $\beta_{3, t, l}=\lambda(G)=\lambda(G, \vec{x})$ we must have $\lambda(\tilde{G}, \vec{x}) \leq \lambda(G, \vec{x})$. Comparing terms in $\lambda(G, \vec{x})$ and $\lambda(\tilde{G}, \vec{x})$ this implies that

$$
\left(\begin{array}{c}
a_{j} \\
2
\end{array}\right) x_{i} x_{j}^{2} \geq\left(\begin{array}{c}
a_{i}-1 \\
2
\end{array}\right) x_{i}^{3} .
$$

In particular, since $x_{i}, x_{j}>0$, we have $x_{i}<x_{j}$.

We give a new weighting $\vec{y}$ for $\tilde{G}$ by setting

$$
y_{v}= \begin{cases}a_{i} x_{i} /\left(a_{i}-1\right), & v \in V_{i} \\ a_{j} x_{j} /\left(a_{j}+1\right), & v \in V_{j}, \\ x_{k}, & v \in V_{k} \text { and } k \neq i, j\end{cases}
$$

It is easy to check that $\vec{y} \in \mathbb{S}_{l}$ is a legal weighting for $\tilde{G}$. We will derive a contradiction by showing that $\lambda(\tilde{G}) \geq \lambda(\tilde{G}, \vec{y})>\lambda(G, \vec{x})=\lambda(G)$.

If $w=a_{i} x_{i}+a_{j} x_{j}=\left(a_{i}-1\right) y_{i}+\left(a_{j}+1\right) y_{j}$ then

$$
\begin{aligned}
\lambda(\tilde{G}, \vec{y})-\lambda(G, \vec{x})= & (1-w)\left(\left(\begin{array}{c}
a_{i}-1 \\
2
\end{array}\right) y_{i}^{2}+\left(\begin{array}{c}
a_{j}+1 \\
2
\end{array}\right) y_{j}^{2}+\left(a_{i}-1\right)\left(a_{j}+1\right) y_{i} y_{j}\right. \\
& \left.-\left(\begin{array}{c}
a_{i} \\
2
\end{array}\right) x_{i}^{2}-\left(\begin{array}{c}
a_{j} \\
2
\end{array}\right) x_{j}^{2}-a_{i} a_{j} x_{i} x_{j}\right)+\left(\begin{array}{c}
a_{i}-1 \\
2
\end{array}\right)\left(a_{j}+1\right) y_{i}^{2} y_{j}+ \\
& \left(\begin{array}{c}
a_{j}+1 \\
2
\end{array}\right)\left(a_{i}-1\right) y_{i} y_{j}^{2}-\left(\begin{array}{c}
a_{i} \\
2
\end{array}\right) a_{j} x_{i}^{2} x_{j}-\left(\begin{array}{c}
a_{j} \\
2
\end{array}\right) a_{i} x_{i} x_{j}^{2} \\
= & \frac{(1-w)}{2}\left(\frac{a_{j} x_{j}^{2}}{a_{j}+1}-\frac{a_{i} x_{i}^{2}}{a_{i}-1}\right)+\frac{a_{i} a_{j} x_{i} x_{j}}{2}\left(\frac{x_{j}}{a_{j}+1}-\frac{x_{i}}{a_{i}-1}\right) .
\end{aligned}
$$

Using (4) it is easy to check that this is strictly positive.

Corollary 10. The t-chromatic Turán density can take irrational values.

Proof. We consider $\beta_{3,2,2 k}$ for $k \geq 3$. In fact, we focus on $\beta_{3,2,6}$, the maximum density of a 2-chromatic 3 -graph that contains no copy of $\mathcal{K}_{6}^{(3)}$. By the previous Theorem, this is 6 times the Lagrangian of the 3-graph with vertex set $\left\{a, a^{\prime}, a^{\prime \prime}, b, b^{\prime}\right\}$ and all edges present except $\left\{a, a^{\prime}, a^{\prime \prime}\right\}$. Assigning weight $x$ to the $a$ 's and weight $y$ to the $b$ 's, we must maximize $6\left(6 x^{2} y+3 x y^{2}\right)$ subject to $3 x+2 y=1$ and $0 \leq x \leq 1 / 3$. A short calculation shows that the choice of $x$ that maximizes this expression is $(\sqrt{13}-2) / 9$, and this results in an irrational value for the Lagrangian. Similar computations hold for larger $k$ as well. 


\section{References}

[E64] P. Erdős, On extremal problems of graphs and generalized graphs, Israel J. Math. 2 (1964), 183-190.

[E71] P. Erdős, On some extremal problems on r-graphs, Disc. Math. 1 (1971), 1-6.

[ES66] P. Erdős and M. Simonovits, A limit theorem in graph theory, Studia Sci. Mat. Hung. Acad. 1 (1966), 51-57.

[ES46] P. Erdős and A.H. Stone, On the structure of linear graphs, Bull. Amer. Math. Soc. 52(1946), 1087-1091.

[FR84] P. Frankl and V. Rödl, Hypergraphs do not jump, Combinatorica 4 (1984), 149 159.

[KNS64] G. Katona, T. Nemetz and M. Simonovits, On a problem of Turan in the theory of graphs (in Hungarian) Mat. Lapok 15 (1964), 228-238.

[M07] W. Mantel, Problem 28, Wiskundige Opgaven, 10 (1907), 60-61.

[MS65] T. Motzkin and E. Strauss. Maxima for graphs and a new proof of a theorem of Turan, Canadian Journal of Mathematics, 17 (1965), 533-540.

[M06] D. Mubayi, A hypergraph extension of Turán's theorem, J. Combin. Theory, Ser. B 96 (2006), 122-134.

[T07] J. Talbot, Chromatic Turán problems and a new upper bound for the Turán density of $\mathcal{K}_{4}^{-}$. Europ. J. Comb 28 (2007), 2125-2142.

[T41] P. Turán, On an extremal problem in graph theory, Mat. Fiz. Lapok 48 (1941). 